Topics in Health \& Biomedical Engineering (THBE) 1(1) (2018) 27-28

Contents List available at VOLKSON PRESS

Medical and Pharmaceutical Research (MPR)

DOI : http://doi.org/10.26480/wcmpr.01.2018.27.28

Journal Homepage: http://www.topicsonbiomed.com//

\title{
SYNDROME DIFFERENTIATION AND TREATMENT OF POSTPARTUM RHEUMATISM
}

\author{
Zhou Zipeng ${ }^{1,2 *}$, Zheng Fuzeng ${ }^{1,2}$, Meng Qingliang ${ }^{1,2}$ \\ ${ }^{1}$ HenanProvince Hospital of Traditional Chinese Medicine, 450002.Zhengzhou, 450000, Henan, P. R. China. \\ ${ }^{2}$ The Second Affiliated Hospital of Henan University of Traditional Chinese Medicine, 450000, Henan, P. R. China. \\ *Corresponding Author Email: 13613863247@163.com
}

This is an open access article distributed under the Creative Commons Attribution License, which permits unrestricted use, distribution, and reproduction in any medium, provided the original work is properly cited

\section{ARTICLE DETAILS}

\section{ARTICLE HISTORY:}

Received 2nd January 2018

Accepted 2nd January 2018

Available online $3^{\text {rd January }} 2018$

\section{KEYWORDS:}

Postpartum rheumatism, TCM syndrome differentiation, Chinese medicine treatment, clinical treatment.

\section{ABSTRACT}

Postpartum rheumatism in postpartum, especially puerperium high incidence, is a common practice in clinical practice. Postpartum rheumatism manifested as joint and systemic symptoms, feelings of different evils or etiology of the symptoms are different. Modern medicine on this lack of knowledge, Chinese medicine treatment of postpartum rheumatism has a unique advantage, a variety of postpartum rheumatoid symptoms, clinical syndrome complex. This article through the clinical practice of Chinese medicine experts on the postpartum rheumatism of the discussion, from the clinical manifestations, pathogenesis and TCM differentiation, Chinese medicine treatment and other aspects of postpartum rheumatism research and further study to provide ideas for further improvement in clinical practice Lay the foundation for the efficacy of postpartum rheumatism.

\section{INTRODUCTION}

Postpartum rheumatism is a woman due to postpartum physically weak, complex feelings of cold and dampness, take the invasion of the flow of muscle, tendons, joints and the emergence of physical and sore joints, pain, numbness, evil wind, cold or mild function limited as a major manifestation of a disease $[1,2]$. The disease is a common cause of postpartum women, frequently disease. Although the disease has symptoms, but no clear tissue damage, no abnormal test indicators, so the existence of Western medicine on the existence of this disease has been doubt, more than the diagnosis of fibromyalgia syndrome. That its puberty depression Disease related. There is no ideal and effective treatment, mostly symptomatic treatment, and add calcium, vitamins, etc., the effect is not significant or disease lingering refractory, patients with poor compliance. Chinese medicine on the syndrome differentiation and treatment has a more comprehensive understanding of the disease that the basis of blood deficiency, or kidney injury, or exogenous cold, or blood stagnation, or mixed with. Is the virtual standard of the waiting [3].

\section{MAJOR SYMPTOMS}

Postpartum rheumatism is mainly manifested as joint and systemic symptoms. Postpartum rheumatism can be involved in the body joints, detailed records, including the waist, knee, hip, etc., and pain, including tingling, by the increase, pain hi press, sore numbness, back tough, unable to pitch, hand foot tendon spine, also recorded the patient's sense of cold is headache nasal congestion aversion to the systemic symptoms [4].

Endocrine factors: women during pregnancy, endocrine will be significant changes. Such as the placenta produced by the chorionic gonadotropin and corticotropin-like anti-inflammatory anti-allergic effect, during pregnancy will produce more than three times the normal amount, and postpartum hormone levels decreased rapidly, unable to meet the needs of the body tissue, resulting in Dysfunction, pain [5]. In addition, during pregnancy, corpus luteum and placental secretion of relaxin, in the pregnant women's pelvic ligament relaxation and flexibility at the same time, the impact of the whole-body ligament, this physiological change, postpartum and cannot be quickly restored, leading to joint Stability is reduced, joint mobility disorders, muscle fatigue [6].
When the joint activities, the ligaments and joints due to abnormal involvement caused by pain.

Immune function factors: during pregnancy due to the role of various specific immunosuppressive factors, immune function is inhibited, and after childbirth, due to the lifting of environmental factors, can lead to disease [7].

Calcium deficiency: maternal during pregnancy for the development of the fetus to provide calcium, phosphorus and other nutrients, the vast majority of calcium from the mother bone tissue mobilization, so that the mother's bone tissue in the calcium deficiency state, resulting in joint pain [8].

Maternal physical exertion: postpartum mothers themselves physical exertion, combined with feeding care baby, day and night labor, joint muscle pain symptoms.

\section{CAUSES OF ONSET AND TCM SYNDROME DIFFERENTIATION}

The cause of the disease and the mechanism is not yet clear, postpartum rheumatism incidence of postpartum or postoperative inadvertently living, washing too cold, clothing is poor, sleep lying, living in wet or indoor air conditioning, air conditioning, fan use improper and other incentives. Whether the cause of the disease is clear and the patient's physical factors, the body can only be a strong description of the reasons, a small part of the weak body cannot say the cause of weakness. Because of the clinical characteristics of the disease in addition to physical discomfort outside the lack of biological function abnormal indicators, the application of modern medical theory cannot explain the pathogenesis of the disease, the relevant research is blank. Chinese medicine for postpartum rheumatism pathogenesis have a clear understanding: women in October pregnant, need a lot of blood to give birth to the fetus, once childbirth, blood loss caused by postpartum qi and blood deficiency, limb joints, muscle bones lost in Ru Yang, Evil take the virtual invasion of the human body, leading to yangbi blocking resistance, gas machine not, meridian barrier, poor blood. Not through the pain, not Rong is Ma, wet resistance is acid swollen, yang lost in the warmth is afraid of the wind cold.

Lack of blood: the woman to the blood-based, for the gas for the use, by, pregnancy, production, milk is dependent on blood filling, if the 
congenital blood deficiency, combined with postpartum blood loss, or postpartum loss is not complex, blood Not by the gas is not exhausted, meridian joints lost in Ru Yang, causing joint muscle pain, numbness.

Kidney qi deficiency: red for the sea of blood, any main child, red any secondary veins are from the cells, are kidney, female by, with, fetal, and are closely related with the kidney. Production injury kidney qi, waist for the kidney of the House, knee is kidney, heel for the kidney by the kidney of the delicate gas deficiency will appear waist and knee pain, leg fatigue or heel pain symptoms.

Evil invasion: postpartum 100 pulse empties, shy reason is not dense, muscle table failure, if the habit of inadvertent, caused by cold dampness evil swoop, missed joints, limbs, affect the normal operation of blood, fatigue and pain

Blood stagnation: gas for the handsome blood, blood for the mother of gas. Blood deficiency is the source, Qi is to promote weakness, or due to cold off the passenger, or due to qi stagnation and poor blood, or because of postpartum trauma, lochia endless, blood addiction into, tired in the passage, blocking barrier.

\section{TREATMENT METHOD}

Postpartum rheumatism is the most prominent symptoms of limb joint pain, clinically based on its pain and the nature of the different, on the basis of dialectical, targeted selection of drugs, can improve the treatment effect. Upper limb joint pain, plus slices of turmeric, Clematis, Notopterygium; lower extremity joint pain, plus alone live, anti-self; knee pain, plus earthworm, pine; waist joint pain, gassan parasitic, Adverse, plus white mustard in addition to the skin outside the sputum. In addition, the disease for a long time should be based on the temperature, according to the appropriate use of insect drugs, such as the artillery A warbler Xuan Tong organs; scorpion, entertainment clams walking channeling network, remove the evil paralysis of the pain; Ecstasy.

\subsection{Blood deficiency condensate type}

Such as dizziness, fatigue, spontaneous perspiration, palpitations, evil wind cold embolism, pale tongue, thin white or small fur, Pulse thin and weak. Governance to Qi and blood, cold and collaterals for the law. Governance law: Huang Mao $30 \mathrm{~g}$, Guizhi $12 \mathrm{~g}$, white peony $12 \mathrm{~g}$, ginger $10 \mathrm{~g}$, jujube $10 \mathrm{~g}$, Millettia $20 \mathrm{~g}$, angelica $10 \mathrm{~g}$, Atractylodes $10 \mathrm{~g}$, mulberry parasite $10 \mathrm{~g}$, Asarum 6g, Chuanwu 8g, safflower 9g, Licorice $10 \mathrm{~g}$

\subsection{Liver and kidney type}

Symptoms of limb joints muscle pain, numbness, obvious at night, joint flexion and extension, and even tendons spasm, body thinner, Yaoxisuanruan, or accompanied by dizziness, tinnitus, upset, dry mouth, irritability night sweats, insomnia, Constipation, red urine, red tongue, less, pulse breakdown. Governance to Yang Yin by fluid, Rongin Tongluo, blood pain for the law. Treatment of drug composition: Rehmannia 30g, yam 15g, 15 grams of mountain meat, medlar $15 \mathrm{~g}$, was Bodhi $10 \mathrm{~g}$, Salvia $15 \mathrm{~g}$, red white peony $10 \mathrm{~g}$, road through $24 \mathrm{~g}$, hive $6 \mathrm{~g}$, Millettia 30g, thin grass 30g.

\subsection{Kidney deficiency type}

Disease, no pain, shortness of breath, back pain, lower limbs, soft, pale tongue, Thin white fur, pulse thin and weak. Governance is: Wen Yang blood, cold and collaterals. Fang Xuan Yang and soup plus or minus. Ingredients: Huangmao $30 \mathrm{~g}$, Chuan Shao $15 \mathrm{~g}$, Angelica $12 \mathrm{~g}$, Rehmannia $30 \mathrm{~g}$, wind $15 \mathrm{~g}$, gun aconite $6 \mathrm{~g}$, ephedra $6 \mathrm{~g}$, antler $9 \mathrm{~g}$ cinnamon $2 \mathrm{~g}$, white mustard $11 \mathrm{~g}$, medlar $15 \mathrm{~g}$, Achyranthes $12 \mathrm{~g}$, Atractylodes $10 \mathrm{~g}$, licorice $12 \mathrm{~g}$.

\subsection{Stagnation of blood stasis type}

As the postpartum more stasis, bleeding is not net, stay by the meridian, caused by poor blood circulation. Zheng Jian: limb joints, muscle pain, pain at the fixed refusal, such as knife-based card, limb numbness, flexion and extension, and even difficult to move. Looking dark, tongue dark or ecchymosis, petechiae, sublingual veins exposed, pulse string astringent. Governing Law: Huoxue Huayu, qiqi Decoction: Biochemical Decoction: angelica $15 \mathrm{~g}$, Chuanxiong $12 \mathrm{~g}$, peach $6 \mathrm{~g}$, safflower $10 \mathrm{~g}$, ginger $12 \mathrm{~g}$, Zhigancao $6 \mathrm{~g}$, Millettia $30 \mathrm{~g}$, green wind rattan $12 \mathrm{~g}$.

\subsection{Lack of blood type}

Due to the weak body, and postpartum Qi deficiency of blood, limbs bivalve darling caused. Symptoms: joint, muscle soreness, after the activities of exacerbated, or limb numbness, gluttony whistle, less fatigue, spontaneous perspiration, palpitations, dizziness, face Huang Shaohua, and even fainting, pale tongue, thin white fur thin pulse Governing Law: Qi and blood, active Quxie. Prescription: bracelet Bi soup addition or subtraction or Huang Mao Guizhi Wuwu Decoction. Angelica $10 \mathrm{~g}$, Chuan Shao $10 \mathrm{~g}$, white peony root $15 \mathrm{~g}$, Rehmannia glutinosa $12 \mathrm{~g}$, raw Huangmao $20 \mathrm{~g}$, Atractylodes $10 \mathrm{~g}$, was $10 \mathrm{~g}$, Zhigancao $6 \mathrm{~g}$, Notopterygium $12 \mathrm{~g}$, independent live $10 \mathrm{~g}$, Guizhi $12 \mathrm{~g}$, Qin Di $15 \mathrm{~g}$, sea wind rattan 15g, wood Fragrant $6 \mathrm{~g}$, Ligustrum lucidum $20 \mathrm{~g}$, Eclipta $15 \mathrm{~g}$

\subsection{Cold dampness resistance type}

Symptoms see the whole-body pain swelling, soreness, heavy, skin numbness, flexion and extension adverse, lower limbs obvious, fear of fear of cold, tired body fatigue, accompanied by less appetite, then pond and cool, pale tongue, Pulse weak or moisten. Weight can be due to pain all night, the degree of joint changes often due to mood swings, or channeling pain (like wind $\mathrm{Bi}$ ) more companion cold fear cold (like cold paralysis) or with upset, irritability, dry mouth, mouth bitter (Like hot $\mathrm{Bi}$ ), chest tightness, headache, frequent heating, abdominal distension, pale tongue, thin white, pulse string. Governing Law: Shugan Jieyu, qi pain. Prescription: Danmie Xiaoyaosan addition and subtraction. $10 \mathrm{~g}$, $10 \mathrm{~g}, 10 \mathrm{~g}, 10 \mathrm{~g}$, etc., $15 \mathrm{~g}$, Atractylodes $10 \mathrm{~g}$, jujube $15 \mathrm{~g}$, licorice $10 \mathrm{~g}$, Polygalaceae $10 \mathrm{~g}$.

\section{CONCLUSIONS}

Postpartum rheumatism for the maternal during the puerperium due to living inadvertently, sweating when the wind, or postpartum physically weak complex and tired, or emotional reasons, there are limbs or joints sorrow and grief, pain, numbness, heavy and a series of symptoms. As the disease although the exact symptoms, but no laboratory indicators and changes in the appearance of the joints, the presence of such diseases have been suffering from Western medicine controversy, so patients often misunderstood, in the treatment method is also more limited. Postpartum rheumatism in postpartum, especially puerperium high incidence, is a common practice in clinical practice. Modern medicine on this lack of knowledge, Chinese medicine treatment of postpartum rheumatism has a unique advantage, a variety of postpartum rheumatoid symptoms, clinical syndrome complex. Through the review of the clinical practice of Chinese medicine experts on the postpartum rheumatoid discussion, from the etiology and pathogenesis, classification treatment, to paralysis, clinical problems to be solved, postpartum rheumatism research status and further study to provide ideas, in order to further improve the clinical practice to lay the foundation for the efficacy of postpartum rheumatism.

\section{REFERENCES}

[1] Yuming, W. 2012. Understanding of postpartum rheumatism [J]. Rheumatology and arthritis, 1 (1), 54-56

[2] Xuewei, L., Sha, W.S., Bin, W. 2016. Postpartum rheumatism of traditional Chinese medicine treatment progress [J]. Chinese folk medicine, 25 (18), 24-27.

[3] Chengquan, Y., Zuwu, Y. 2013. Postpartum rheumatism dialectical treatment [J]. Chinese National Folk Medicine, 129.

[4] Biao, M., Lizhen, G. 2013. Zhao Heping treatment of postpartum rheumatism experience $[\mathrm{J}]$. Chinese Journal of Traditional Chinese Medicine, 5 (41), 32

[5] Lihong, Z. 2006. Chinese medicine treatment of postpartum rheumatism ideas and methods $[\mathrm{J}]$. Chinese Journal of National Medicine, 4, 20.

[6] Yuzhen, Z. 2002. Chinese medicine gynecology [M]. Beijing: China Chinese Medicine Publishing House, R7.

[7] Yuming, W. 2014. Postpartum rheumatism characteristics and clinical experience [J]. Sichuan Traditional Chinese Medicine, 32 (1), 59.

[8] Yingze, Z., Xiaoping, Y. 2010. Guizhi A pill treatment of rheumatism in women clinical application [J]. Chinese Journal of Rheumatology, $13(3,4), 400$. 